\title{
Grenzen der Sprache, Grenzen der Sprachwissenschaft: Breslauer Konferenz über ein grenzloses Thema
}

\author{
Blanka Datinská - Markéta Valíčková
}

Kann und darf man die Sprachwissenschaft abgrenzen? Gibt es überhaupt Grenzen, hinter die das Auge eines Linguisten nicht schauen sollte?

Diese und viele andere Fragen wurden vom 14. bis 16. September beim sechsten Jahrgang der internationalen Tagung Linguistische Treffen gestellt und aus verschiedenen Perspektiven beantwortet. 90 Gäste aus 16 Ländern aus der ganzen Welt haben Ergebnisse und Gegenstände ihrer linguistischen Forschungen präsentiert, die mehr oder weniger die nicht genau festgelegten und heutzutage immer loseren Grenzen der Sprachwissenschaft diskutiert haben. Im Rahmen des Programms wurde nicht nur die deutsche Gegenwartssprache, sondern auch der kontrastive Vergleich zwischen dem Deutschen und Polnischen, Dänischen oder Litauischen besprochen. Neben den fachlich orientierten Diskussionen nach den einzelnen Vorträgen kam es in den Kaffeepausen auch zum Meinungs- und Erfahrungsaustausch, die für uns als Einsteigerinnen in die internationale Forschungssphäre von großer Bedeutung waren.

Die Konferenzbeiträge wurden in vier thematische Sektionen eingeteilt: Sprache im öffentlichen Raum, Sprechen und Kommunizieren im Vergleich, Konvention und Innovation im Interagieren linguistisch erfassen und Varietäten. Sozio- und diskurslinguistische Themen wie Grenzen der Hass-Sprache in sozialen Netzwerken, Selbstdarstellung auf Twitter als Bestandteil der Online-Identität, Analyse der Online-Kommunikation, sprachliche Grenzen in Radikalisierungsprozessen oder Manipulation und Kommunikation durch Medien waren genauso interessant wie die Referate, die korpuslinguistische Fragen und übersetzungswissenschaftliche Phänomene angedeutet haben. Die Grenzen zwischen den einzelnen Sektionen wurden aber auch überschritten, sowie die Grenzen zwischen den linguistischen Teildisziplinen.

Ins Tagungsprogramm wurden auch zwei Plenarvorträge von Prof. Dr. Hab. Michaił Kotin und Doz. RNDr. Tomáš Hoskovec, CSc. eingereiht, die sowohl einen geschichtsorientierten, als auch einen kritischen Blick auf die Sprache und Sprachwissenschaft geworfen haben. Unsere Referate wurden in der vierten Sektion „Varietäten“ vorgetragen. Kurz 
nacheinander haben wir unsere Forschungsthemen vorgestellt: korpusbasierte Untersuchung am Beispiel des Verbs ,lassen' und die Movierung und Geschlechtsspezifikation in den Berufsbezeichnungen in der deutschen Gegenwartssprache. Mit einer translatologischen und einer ein bisschen provokanten Thematik haben wir zu einer gedankenreichen zweitägigen Diskussion beigetragen, die dann mit einer nicht ausgesprochenen Schlussfolgerung beendet wurde - Grenzen der Sprache, Grenzen meiner Welt, grenzenlose Sprachwissenschaft.

Mgr. Blanka Datinská / blanka.datinska@mail.muni.cz

Mgr. Markéta Valíčková / marketa.valickova@mail.muni.cz

Masarykova univerzita, Filozofická fakulta, Ústav germanistiky, nordistiky a nederlandistiky Arne Nováka 1, 60200 Brno, CZ 